\title{
Post-mortem Redistribution of Alprazolam in Rats
}

\author{
Jana Hořínková1, Petr Kozlík'², Tomáš Kř́žek ${ }^{2}$, \\ Danica Michaličková ${ }^{1}$, Martin Šíma ${ }^{1}$, Ondřej Slanař ${ }^{1}$ \\ ${ }^{1}$ Institute of Pharmacology, First Faculty of Medicine, Charles University \\ and General University Hospital in Prague, Prague, Czech Republic; \\ ${ }^{2}$ Department of Analytical Chemistry, Faculty of Science, Charles University, \\ Prague, Czech Republic
}

Received March 7, 2020; Accepted November 2, 2020.

Key words: Alprazolam - Benzodiazepines - Overdose - Post-mortem redistribution - Animal model - Forensic toxicology

\begin{abstract}
The post-mortem toxicological findings may be misinterpreted, if the drug undergoes substantial post-mortem redistribution. As alprazolam is one of the most frequently evaluated drug for legal/forensic reasons in drug-related fatalities, we studied possible changes in alprazolam distribution after death in a rat model. Rats were sacrificed 30 minutes after alprazolam administration. Blood and tissue samples from 8 animals per sampling time were collected at 0, 2, 6, and $24 \mathrm{~h}$ after death. The experimental samples were assayed for alprazolam using validated UHPLC-PDA method. Median blood alprazolam concentrations increased approximately 2 times compared with ante-mortem levels due to the redistribution during early post-mortem phase and then slowly decreased with a half-life of $60.7 \mathrm{~h}$. The highest alprazolam tissue concentrations were found in fat and liver and the lowest levels were observed in lungs and brain. The median amount of alprazolam deposited in the lungs was relatively stable over the 24-h post-mortem period, while in heart, liver and kidney the deposited proportion of administered dose increased by $43-48 \%$ in comparison with ante-mortem values indicating continuous accumulation of alprazolam into these tissues. These results provide evidence needed for the interpretation of toxicological results in alprazolam-related fatalities and demonstrate modest alprazolam post-mortem redistribution.
\end{abstract}

This study was supported by the Charles University Project Progres Q25, Research Centre program No. UNCE/SCI/014, and a grant No. SVV 260523.

Mailing Address: Martin Šíma, PharmDr., PhD., Institute of Pharmacology, First Faculty of Medicine, Charles University and General University Hospital in Prague, Albertov 4, 12800 Prague 2, Czech Republic; Phone: +420 224968 161; e-mail: martin.sima@lf1.cuni.cz 


\section{Introduction}

Drug overdose belongs among common causes of death worldwide with mortality rate of approximately 22.6 and 217 cases per million inhabitants in the EU and USA, respectively (European Monitoring Centre for Drugs and Drug Addiction, 2019). Although the majority of drug-related deaths in Europe occur in men, suicidal drug overdoses are more frequent in women. Most fatal drug overdoses are linked to the use of opioids, central nervous system depressants including benzodiazepines or polydrug use. Alprazolam itself was the fifth most common drug detected in 6,209 drug-related deaths in the USA in 2016 (Hilberg et al., 1999). Alprazolam is therefore frequently evaluated for legal/forensic reasons in drug-related fatalities.

The post-mortem toxicological findings may be misinterpreted if the drug undergoes substantial post-mortem redistribution, which is mainly expected for basic, lipophilic compounds widely distributed into peripheral compartments (Kugelberg et al., 2004; Castaing et al., 2006). The mechanisms of post-mortem redistribution have not been fully characterized, but leakage of intracellular content after cellular death (Musther et al., 2014), disruption of physiological barriers (Banks et al., 1992), degradation of the compounds by gut microorganisms and their diffusion from gastrointestinal content into the body tissues belong among known causes contributing to this phenomenon (European Monitoring Centre for Drugs and Drug Addiction, 2019). Moreover, metabolism may also continue during the first few hours after death (Jonsson et al., 2004).

Post-mortem redistribution has been described for number of drugs involved in fatal drug-related overdoses including haloperidol, morphine, citalopram, triazolam or diazepam (Koren and MacLeod, 1985; Shiota et al., 2004; Kposowa and McElvain, 2006).

Alprazolam with log $\mathrm{P}$ of 2.12 belongs among lipophilic compounds; it has apparent volume of distribution of approximately $1 \mathrm{I} / \mathrm{kg}$ in man (Greenblatt and Wright, 1993). These characteristics suggest possible role of post-mortem redistribution for this drug. Reports that evaluated central to peripheral blood concentrations ratios in human have suggested that alprazolam may exhibit a modest post-mortem redistribution (Hargrove and McCutcheon, 2008; Han et al., 2012). However, there are no data on post-mortem redistribution from direct measurement of alprazolam concentrations in particular tissues available. Therefore, the aim of our study was to describe possible changes in alprazolam distribution after death in rats.

\section{Methods}

Chemicals

Alprazolam was purchased from Sigma-Aldrich (Saint Louis, USA). The drug was then dissolved in a mixture of $70 \%$ ethanol and saline $(1: 1 ; \mathrm{v} / \mathrm{v})$ for the purpose of intraperitoneal application. Isoflurane was used as IsoFlo $250 \mathrm{ml}$ (Zoetis, Parsippany, USA). 


\section{Animals}

Female Wistar rats (Velaz, Prague, Czech Republic) were used throughout the study. They were maintained under standard conditions (12-h light-dark cycle, $22 \pm 2{ }^{\circ} \mathrm{C}$ temperature and $50 \pm 10 \%$ relative humidity) and fed on water and standard granulated diet ad libitum. All experiments were performed in accordance with the Guiding Principles in the Use of Animals in Charles University, First Faculty of Medicine, and every effort was made to minimize animal suffering. The experimental animal project was approved by the Ministry of Education, Youth and Sports of the Czech Republic under the number MSMT-9445/2018-8.

\section{Experimental procedure}

Rats were randomly divided into four groups: (1) immediate autopsy, (2) autopsy 2 hours after death, (3) autopsy 6 hours after death, and (4) autopsy 24 hours after death. All animals were anesthetized by inhalation of $2-5 \%$ isoflurane and anaesthesia was maintained throughout the procedure. Following anesthetization, rats were injected alprazolam intraperitoneally at a dose of $4 \mathrm{mg}$ or $6 \mathrm{mg}$ for animals weighting $\leq 250$ g or more, respectively. Thirty minutes after alprazolam administration, an ante-mortem blood sample was taken via cardiac puncture from each rat. Immediately following the sampling, rats were sacrificed by cervical dislocation and death was confirmed by the lack of a heartbeat. Following death, rats in groups $2-4$ were left lying on their backs at room temperature $\left(22 \pm 2{ }^{\circ} \mathrm{C}\right)$ for the defined time to autopsy. Post-mortem aortic blood samples were drawn and the following tissue samples were also collected at the time of autopsy: liver (left lateral lobe), left kidney, heart, left lung, brain and abdominal fat. The tissue samples were cleaned with tissue paper, weighted and immediately homogenized with Tissue-Tearor homogenizer model 985-370 (BioSpec Products, Inc., Bartlesville, USA) in two volumes of $80 \%$ acetonitrile and then vortexed for $30 \mathrm{~s}$. Both blood samples and tissue homogenates were centrifuged for 10 minutes at $2,500 \times \mathrm{g}$ $\left(4^{\circ} \mathrm{C}\right)$. Blood samples and tissue supernatants were then stored at $-80{ }^{\circ} \mathrm{C}$ before further processing. Before the chromatography analysis, the blood samples and tissue supernatants were deproteinized by acetonitrile, adding $60 \mu \mathrm{l}$ of $100 \%$ acetonitrile to $20 \mu \mathrm{l}$ of sample and performing deproteinization in an Eppendorf tube by vortexing for $15 \mathrm{~s}$. Then, both blood and tissue samples were centrifuged at $16,500 \times \mathrm{g}$ for $6 \mathrm{~min}$, and $50 \mu \mathrm{l}$ of supernatant was transferred into LC vials.

\section{Analysis of alprazolam}

Determination of alprazolam in different tissues and blood samples was carried out using Acquity UPLC H-class equipment (Waters Corporation, Milford, MA). LC column Poroshell HPH C18 (3.00 mm i.d. × 100 mm, $2.7 \mu \mathrm{m})$ from Agilent Technologies (Waldbronn, Germany), thermostatted at $30{ }^{\circ} \mathrm{C}$, was used for the analysis. The mobile phase consisted of $10 \mathrm{mM}$ ammonium phosphate, $\mathrm{pH} 2.80$ (Solvent A) and acetonitrile (Solvent B). The flow rate of the mobile phase 
was maintained at $0.5 \mathrm{ml} / \mathrm{min}$. The optimized gradient program ( $\mathrm{min} / \% \mathrm{~B}$ ) was 0/30,1/30, 3.5/60,4/80,7/80,7.5/30, and 10/30. The injection volume was $1 \mu \mathrm{l}$, and samples were kept at $10^{\circ} \mathrm{C}$. Detection was performed by diode array detector, and the wavelength was set to $245 \mathrm{~nm}$.

The method was validated according to the FDA guidance on analytical procedures and method validation to demonstrate that it is suitable for its intended purpose (U. S. Food and Drug Administration, 2015). The selectivity of the method was verified by mass spectrometry operated in the scan mode (Triple Quad 6460 mass spectrometer; Agilent Technologies, Waldbronn, Germany).

Selectivity was monitored by injecting all studied tissue extracts. These chromatograms showed no interfering compound (no $\mathrm{m} / \mathrm{z}$ was observed except $\mathrm{m} / \mathrm{z}$ corresponding to alprazolam) within the retention time window of alprazolam. Moreover, the DAD peak purity test of all analysed samples was successfully met for the alprazolam peak, which ensures high selectivity. Selectivity was thus confirmed independently by DAD and mass spectrometry. The calibration curve was constructed in the $80 \%$ acetonitrile with nine concentration levels $(0.1 ; 0.2$; $0.5 ; 1 ; 2 ; 5 ; 10 ; 50 ; 100 \mu \mathrm{g} / \mathrm{ml})$ by plotting the peak area of alprazolam against its concentration. Calibration was performed before each batch of samples. Standard plots were constructed and linearity was evaluated statistically by linear regression analysis using the least-squares regression method. To confirm the reliability of our results derived from the calibration curve, we performed determination using the standard addition method with selected samples of different tissue extracts and blood. The standard was added into blood and tissue extract samples before deproteinization to include the potential effect of deproteinization. No significant difference in concentration means was observed between results obtained using both methods. This result proved that tissue and blood matrices have no effect on the reliable quantification of alprazolam. The linearity was evaluated through the calibrations providing coefficients of determination $\left(R^{2}\right)$ higher than 0.9997 which indicate excellent linearity. Limit of detection value was $0.02 \mu \mathrm{g} / \mathrm{ml}$, determined as $3.3 \times \sigma / S$ ratio, where $\sigma$ is the highest baseline noise obtained from the blank blood/ tissue extracts, and $\mathrm{S}$ is the slope of the regression line (based on peak heights). Limit of quantification was the lowest point of the calibration $(0.1 \mu \mathrm{g} / \mathrm{ml})$.

Method accuracy and precision were evaluated by measuring 5 replicates at four different concentrations $(0.1 ; 1 ; 5$, and $50 \mu \mathrm{g} / \mathrm{ml})$ prepared by spiking alprazolam into blank tissue extracts and blood. The accuracy (relative error, \%) was within $\pm 3.0 \%$, and the inter- and intra-day precisions (RSD, \%) were within $\pm 2.5 \%$. These samples were also used as quality control (QC) samples. QC samples were injected after each $7^{\text {th }}$ sample to assess the validity of the analytical method. Recovery was evaluated by comparing the area of the alprazolam standard peak of the pre-proteinprecipitation spiked blood sample with that of the corresponding post-proteinprecipitation spiked sample at three concentrations $(0.1 ; 10$, and $50 \mu \mathrm{g} / \mathrm{ml})$. Since there is no reference material of different tissues containing alprazolam, the recovery 
was simulated by fortifying of different tissue homogenates with the standard of alprazolam at three concentrations $(0.1 ; 10$, and $50 \mu \mathrm{g} / \mathrm{ml})$. Extraction and protein precipitation was performed with $80 \%$ acetonitrile and vortexed for $30 \mathrm{~s}$. The recovery ranged from 96.8 to $100.9 \%$.

\section{Data analysis and statistics}

Tissue concentrations $(\mu \mathrm{g} / \mathrm{g}$ ) were calculated from measured supernatant concentrations $(\mu \mathrm{g} / \mathrm{ml})$, used amounts of solvent $(\mathrm{ml})$ and weights of homogenized tissues $(\mathrm{g})$. Subsequently, both tissue and blood concentrations were normalized per dose of $20 \mathrm{mg} / \mathrm{kg}$.

Distribution half-life was calculated as $(t \times \ln 2) / \ln \left(C_{2} / C_{24}\right)$, where $C_{2}$ and $C_{24}$ are median alprazolam blood concentration 2 and $24 \mathrm{~h}$ after death, respectively, and $\mathrm{t}$ is $22 \mathrm{~h}$ as the time between $\mathrm{C}_{2}$ and $\mathrm{C}_{24}$.

Median and interquartile range (IQR) values were calculated using MS Excel 2010 (Microsoft Corporation, Redmond, USA). Significance of differences in alprazolam concentrations/tissue distribution between group 1 and the other groups was determined by the Mann-Whitney test, while potential differences in weights and weight-normalized doses between study groups were examined by the Kruscal-Wallis test using GraphPad Prism 8.2.1 (GraphPad Software, Inc., La Jolla, USA). Statistical significance was considered at $p \leq 0.05$.

\section{Results}

Thirty-two female rats weighing 162-365 g were enrolled in this study; eight rats per group. Administered alprazolam doses ranged from 16.4 to $24.7 \mathrm{mg} / \mathrm{kg}$. There were no significant differences in animal weight $(p=0.97)$ or administered alprazolam doses $(p=0.96)$ between study groups.

Post-mortem changes in dose-normalized blood and tissue concentrations of alprazolam are presented in Figure 1. All post-mortem groups showed significantly increased blood drug concentrations compared with ante-mortem alprazolam blood levels. The ratio between median post-mortem and ante-mortem alprazolam blood concentrations were 1.78, 1.54 and 1.39 at 2, 6 and 24 hours after death, respectively. Thus, steep rise in alprazolam blood levels during early post-mortem phase was followed by slow decrease with a half-life of $60.7 \mathrm{~h}$.

Distribution of alprazolam into the tissues expressed as percentages of the total administered alprazolam dose are showed in Table 1.

\section{Discussion}

This study was designed to describe possible post-mortem alprazolam redistribution in rat in order to improve our understanding and interpretation of the toxicological findings in alprazolam related fatalities that belong among frequent intentional drug overdoses world-wide. We conducted the study in female rats, since women are 4 times more likely to die from drug poisoning than men (Kposowa and McElvain, 
Liver

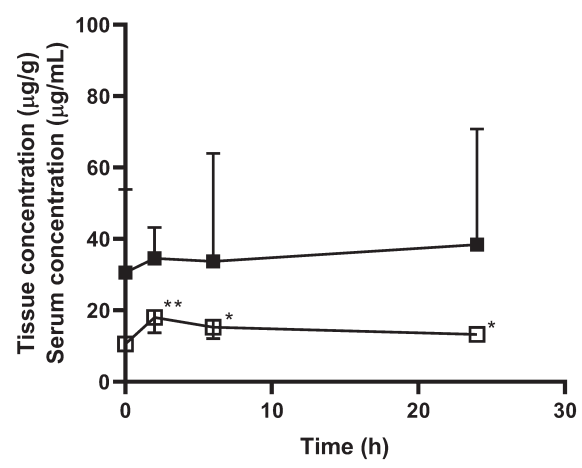

Kidney

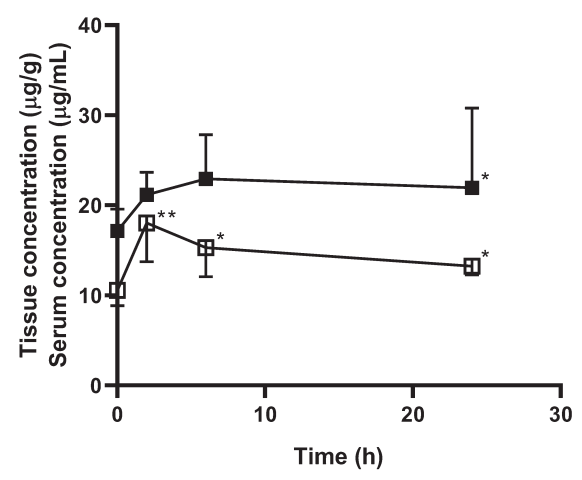

Lung

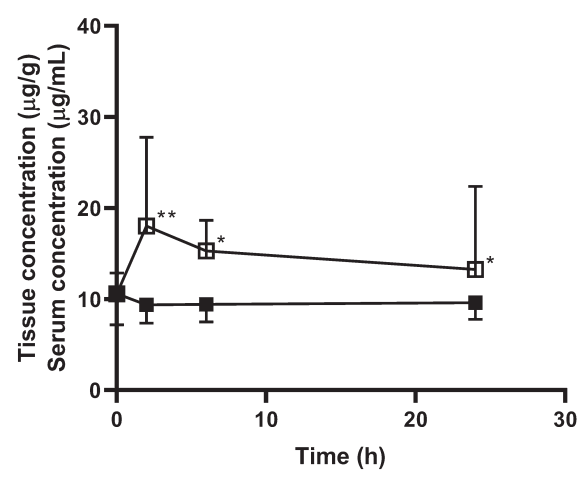

Fat

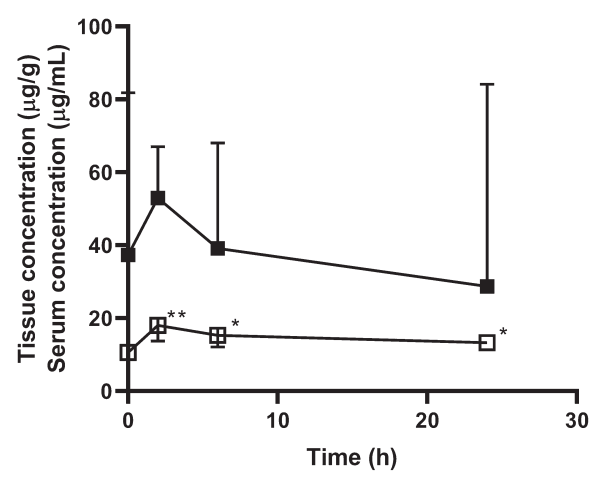

Heart

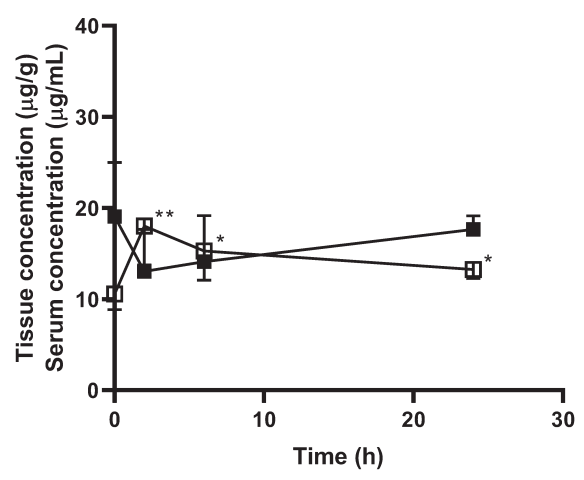

Brain

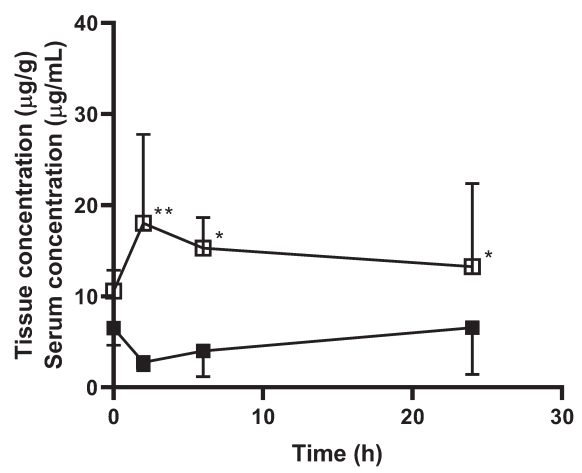

¥ Serum concentration

Figure 1 - Post-mortem changes of alprazolam concentrations normalized per dose of $20 \mathrm{mg} / \mathrm{kg}$ in the rat blood and tissues. Values are represented as median (interquartile range); $n=8 ; *<<0.05 ; * *<<0.005$ vs. 0 h after death. 


\begin{tabular}{|c|c|c|c|c|}
\hline & $\begin{array}{c}\text { Group } 1 \\
0 \mathrm{~h}\end{array}$ & $\begin{array}{c}\text { Group } 2 \\
2 \mathrm{~h}\end{array}$ & $\begin{array}{c}\text { Group } 3 \\
6 \mathrm{~h}\end{array}$ & $\begin{array}{c}\text { Group } 4 \\
24 \text { h }\end{array}$ \\
\hline Liver & 5.17 & 7.11 & $8.17^{*}$ & 7.38 \\
\hline Kidney (left) & 0.29 & 0.34 & 0.42 & $0.43 *$ \\
\hline Heart & 0.21 & 0.22 & 0.23 & 0.32 \\
\hline Lung (left) & 0.08 & 0.08 & 0.09 & 0.10 \\
\hline Brain & 0.32 & 0.10 & 0.14 & 0.24 \\
\hline
\end{tabular}

${ }^{*} \mathrm{p}<0.05$ vs. $0 \mathrm{~h}$ after death

2006). A fix dose of 4 or $6 \mathrm{mg}$ was administered to each animal with respect to its actual body weight in order to reach approximate dose of $20 \mathrm{mg} / \mathrm{kg}$ for each animal, which corresponds to human equivalent dose of $3.3 \mathrm{mg} / \mathrm{kg}$ (rat/human dose conversion factor of 6 has been reported) (Nair and Jacob, 2016). The actual doses ingested are usually unknown in human alprazolam-related fatalities, however, the drug has been reported among compounds with the most frequent toxic levels detected in forensic analyses in drug related fatalities (Jonsson et al., 2004). A study in 131 deliberate alprazolam self-poisoning cases reported that the median (IQR) alprazolam dose was 23 defined daily doses (10-40) (Isbister et al., 2004), which is less in comparison with the dose we used. However, other individual case reports have shown high plasma and tissue levels indicating that the dose ingested must have been considerably higher (Jenkins et al., 1997). Since the doses ingested in an intentional overdose are in reality highly uncertain, we have chosen the human equivalent dose for our study corresponding to the possible worst case scenario for acute intoxication after ingestion of whole alprazolam high strength package (100 tablets, $2 \mathrm{mg}$ ).

Alprazolam blood levels reported for human fatal intoxications are extremely variable. While some repots mention plasma levels within the toxic range of 0.1-0.4 $\mu \mathrm{g} / \mathrm{ml}$ (Jones et al., 2016; Mclntyre et al., 2017), Jenkins et al. (1997) detected plasma levels in a human alprazolam fatality of $2.1-2.3 \mu \mathrm{g} / \mathrm{ml}$ that is approximately $20 \%$ of the ante-mortal levels seen in our study.

We observed significantly increased blood drug concentrations at all sampling times post-mortem. This phenomenon has been previously described for some other lipophilic drugs, e.g. thioridazine, morphine or citalopram (Koren and Klein, 1992; Kugelberg et al., 2004; Castaing et al., 2006). However, the interindividual variability of the drug concentration increase after death is high, in a few animals the post-mortem increase of alprazolam blood levels was as large as 6-fold. This is again comparable to the previous observations for morphine with up to 5 -fold drug level increase (Koren and Klein, 1992). 
The highest alprazolam tissue concentrations were found in fat and liver in group 1 (autopsy 30 min after intraperitoneal administration), whereas the lowest levels were observed in lungs and brain. These findings follow a similar distribution pattern described for ${ }^{14} \mathrm{C}$-alprazolam $30 \mathrm{~min}$ after intravenous administration (Banks et al., 1992) and correspond also to distribution of diazepam and triazolam that both belong among benzodiazepines (Shiota et al., 2004). Although alprazolam concentration in the brain was low, the percentual distribution of the administered dose was similar to that found in left kidney at the time of death (Table 1). The highest percentage of alprazolam dose were accumulated in the liver. It can be expected that largest proportion of alprazolam dose accumulates in adipose tissue, however, as the total body fat is unknown the distribution could not be estimated.

The amount of alprazolam deposited in the lung tissue was relatively stable over the 24-h post-mortem period, while in heart, liver and kidney the deposited proportion of administered dose increased by $43-48 \%$ at $24 \mathrm{~h}$ after death in comparison with pre-mortem values indicating continuous accumulation of alprazolam into these tissues. The tissue concentrations tended to increase correspondingly to the percentage of the dose deposited in liver and kidney, while the drug tissue concentration dropped in the heart early after death contrary to the increasing drug accumulation in the tissue. This was likely caused by an increased intramyocardial water content that may be seen after death especially in females (Boyd and Knight, 1963). On the other hand, both brain alprazolam content and brain tissue concentrations decreased early after death with subsequent tendency to reach equilibrium with blood concentrations. Due to the high variability of tissue concentrations obtained, the differences of tissue concentrations from the post-mortem sample only reached statistical significance for kidney at $24 \mathrm{~h}$.

The clinical data on alprazolam distribution post-mortem are extremely limited. In a single fatal overdose, the ratios between tissue and plasma drug levels in kidney and liver were approximately 1.7 and 4.2, respectively (Jenkins et al., 1997), which well corresponds to the individual tissue to plasma ratios seen in our study ranging from 0.2 to 4.1 for kidney and from 0.9 to 9.2 for liver samples. The anatomical proximity between the different organs in rats in our model thus did not result in unrealistically overestimated post-mortem redistribution values for the human toxicological findings. In case there is an unabsorbed drug in the stomach or gastrointestinal tract at the time of death, this may be redistributed to surrounding tissues (Pounder et al., 1996). In order to eliminate this phenomenon, we administered alprazolam intraperitoneally in this study. Although the most alprazolam overdoses are expected to follow oral ingestion of the drug in humans, the absorption is fast and complete with $\mathrm{T}_{\max }$ of $1 \mathrm{~h}$ and absolute bioavailability 80-100\% (Greenblatt and Wright, 1993). Therefore, substantial alprazolam redistribution from the gastrointestinal tract is not expected in clinical settings. 


\section{Conclusion}

Median central blood alprazolam concentrations increased approximately 1.5-2 times due to the redistribution over the 24-h post-mortem period and there was a substantial variability of the extent of alprazolam redistribution (0.9-6.1 fold).

The median amount of alprazolam deposited in the lung tissue was relatively stable over the 24-h post-mortem period, while in heart, liver and kidney the deposited proportion of administered dose increased by $43-48 \%$ at $24 \mathrm{~h}$ after death in comparison with pre-mortem values indicating continuous accumulation of alprazolam into these tissues.

These results demonstrate modest alprazolam redistribution post-mortem. However, due to the high variability of alprazolam blood and tissue concentrations seen, the estimation of drug exposure or time from death can't be reliably done based on these pharmacokinetic data.

\section{References}

Banks, W. R., Yamakita, H., Digenis, G. A. (1992) Metabolism and distribution of 1-[14C]alprazolam in rats. J. Pharm. Sci. 81, 797-801.

Boyd, E. M., Knight, L. M. (1963) Postmortem shifts in the weight and water levels of body organs. Toxicol. Appl. Pharmacol. 5, 119-128.

Castaing, N., Titier, K., Canal-Raffin, M., Moore, N., Molimard, M. (2006) Postmortem redistribution of two antipsychotic drugs, haloperidol and thioridazine, in the rat. J. Anal. Toxicol. 30, 419-425.

European Monitoring Centre for Drugs and Drug Addiction (EMCDDA) (2019) European Drug Report: Trends and Developments. Available at: http://www.emcdda.europa.eu/system/files/publications /11364/20191724_TDAT19001ENN_PDF.pdf

Greenblatt, D. J., Wright, C. E. (1993) Clinical pharmacokinetics of alprazolam. Therapeutic implications. Clin. Pharmacokinet. 24, 453-471.

Han, E., Kim, E., Hong, H., Jeong, S., Kim, J., In, S., Chung, H., Lee, S. (2012) Evaluation of postmortem redistribution phenomena for commonly encountered drugs. Forensic Sci. Int. 219, 265-271.

Hargrove, V. M., McCutcheon, J. R. (2008) Comparison of drug concentrations taken from clamped and unclamped femoral vessels. J. Anal. Toxicol. 32, 621-625.

Hilberg, T., Ripel, A., Slordal, L., Bjorneboe, A., Morland, J. (1999) The extent of postmortem drug redistribution in a rat model. J. Forensic Sci. 44, 956-962.

Isbister, G. K., O'Regan, L., Sibbritt, D., Whyte, I. M. (2004) Alprazolam is relatively more toxic than other benzodiazepines in overdose. Br. J. Clin. Pharmacol. 58, 88-95.

Jenkins, A. J., Levine, B., Locke, J. L., Smialek, J. E. (1997) A fatality due to alprazolam intoxication. J. Anal. Toxicol. 21, 218-220.

Jones, A. W., Holmgren, A., Ahlner, J. (2016) Post-mortem concentrations of drugs determined in femoral blood in single-drug fatalities compared with multi-drug poisoning deaths. Forensic Sci. Int. 267, 96-103. Jonsson, A., Holmgren, P., Ahlner, J. (2004) Fatal intoxications in a Swedish forensic autopsy material during 1992-2002. Forensic Sci. Int. 143, 53-59.

Koren, G., MacLeod, S. M. (1985) Postmortem redistribution of digoxin in rats. J. Forensic Sci. 30, 92-96. Koren, G., Klein, J. (1992) Postmortem redistribution of morphine in rats. Ther. Drug Monit. 14, 461-463. Kposowa, A. J., McElvain, J. P. (2006) Gender, place, and method of suicide. Soc. Psychiatry Psychiatr. Epidemiol. 41, 435-443. 
Kugelberg, F. C., Druid, H., Carlsson, B., Ahlner, J., Bengtsson, F. (2004) Postmortem redistribution of the enantiomers of citalopram and its metabolites: an experimental study in rats. J. Anal. Toxicol. 28, 631-637.

McIntyre, I. M., Gary, R. D., Joseph, S., Stabley, R. (2017) A fatality related to the synthetic opioid U-47700: Postmortem concentration distribution. J. Anal. Toxicol. 41, 158-160.

Musther, H., Olivares-Morales, A., Hatley, O. J., Liu, B., Rostami Hodjegan, A. (2014) Animal versus human oral drug bioavailability: do they correlate? Eur. J. Pharm. Sci. 57, 280-291.

Nair, A. B., Jacob, S. (2016) A simple practice guide for dose conversion between animals and human. J. Basic Clin. Pharm. 7, 27-31.

Pounder, D. J., Fuke, C., Cox, D. E., Smith, D., Kuroda, N. (1996) Postmortem diffusion of drugs from gastric residue: an experimental study. Am. J. Forensic Med. Pathol. 17, 1-7.

Shiota, H., Nakashima, M., Terazono, H., Sasaki, H., Nishida, K., Nakamura, J., Taniyama, K. (2004) Postmortem changes in tissue concentrations of triazolam and diazepam in rats. Leg. Med. (Tokyo) 6 , 224-232.

U. S. Food and Drug Administration; Department of Health and Human Services (2015) Analytical Procedures and Methods Validation for Drugs and Biologics: Guidance for Industry. Available at: https://www.fda.gov /files/drugs/published/Analytical-Procedures-and-Methods-Validation-for-Drugs-and-Biologics.pdf 